\title{
Tipologías residenciales en comunidades chilenas en condiciones de precariedad habitacional
}

\author{
Soledad Burgos, ${ }^{1}$ Rosalina Jorge Koifman, ${ }^{2}$ \\ Rosa Montaño Espinoza ${ }^{1}$ y Jorge Atria Curi ${ }^{3}$
}

Forma de citar Burgos S, Koifman RJ, Montaño Espinoza R, Atria Curi J. Tipologías residenciales en comunidades chilenas en condiciones de precariedad habitacional. Rev Panam Salud Publica. 2011;29(1): 32-40.

RESUMEN Objetivo. Identificar dimensiones residenciales que caractericen el ambiente físico y social en los campamentos de Chile y construir tipologías que permitan elaborar perfiles con esos atributos residenciales distintivos.

Métodos. Se estudió el universo de campamentos $(\mathrm{n}=122)$ de la Región Metropolitana (Chile) a partir del Catastro Nacional de Campamentos realizado por la fundación "Un Techo para Chile" en 2007. Se recolectó información proveniente de informantes clave sobre las comunidades y los lugares de emplazamiento, variables que fueron modeladas empleando un análisis factorial para identificar dimensiones residenciales, las que a su vez fueron refinadas con la técnica de agrupamientos de k-medias.

Resultados. El análisis factorial destacó tres dimensiones subyacentes: respuesta social local, ambiente extracomunitario y servicios básicos. Las variables centrales en la formación de esas categorías fueron la existencia de proyectos habitacionales, el área urbana y el acceso a electricidad, respectivamente. El análisis de agrupamientos generó cuatro perfiles que combinaron las tres dimensiones: perfil urbano con baja precariedad de servicios básicos $(\mathrm{n}=30)$, perfil urbano-rural con alto potencial de respuesta social local $(\mathrm{n}=32)$, perfil urbano con alta amenaza ambiental $(\mathrm{n}=43)$ y perfil rural con bajo potencial de respuesta social local $(\mathrm{n}=17)$. Conclusiones. Las dimensiones residenciales formadas son consistentes con el modelo teórico revisado y sugieren indicadores relevantes para el seguimiento de estas comunidades. La identificación de perfiles permite dimensionar la heterogeneidad de realidades residenciales contribuyendo a la priorización de dominios de déficit o riesgos que pueden estar presentes en cada grupo, para con ello profundizar en su investigación y oportunidades de acción.

Palabras clave Asentamientos humanos; áreas de pobreza; comunidades vulnerables; censos; salud ambiental; organización social; organización comunitaria; Chile.

La precariedad habitacional se encuentra entre las principales causas de deterioro de la calidad de vida en las pobla-

1 Universidad de Chile, Facultad de Medicina, Escuela de Salud Pública, Santiago, Chile. La correspondencia se debe dirigir a Soledad Burgos, sburgos@med.uchile.cl

2 Fundação Oswaldo Cruz, Escola Nacional de Saúde Pública Sergio Arouca, Rio de Janeiro, Brasil.

3 Pontificia Universidad Católica, Instituto de Sociología, Santiago, Chile. ciones más pobres (1). En América Latina y el Caribe se estima que cerca de $32 \%$ de la población urbana reside en condiciones de alta informalidad, en viviendas ubicadas en zonas de riesgo ambiental e insuficiente infraestructura y servicios $(1,2)$. En el caso de Chile, estas poblaciones residen en "campamentos", comunidades de ocho o más familias sin acceso regular a alcantarillado, agua potable o energía eléctrica, y en situación irregular en cuanto a la tenencia del terreno (3). Según estimaciones censales de 2007, en todo el país hay 533 campamentos — cerca de 29000 familias-, cuyo ambiente residencial ha sido escasamente abordado para analizar impactos potenciales en la salud de estos residentes (3).

Varios estudios llevados a cabo en países desarrollados, al comparar zonas con 
distintos niveles de carencias, revelan que la calidad del ambiente residencial -en términos de servicios, recursos, apoyo social y estructura socioeconómica- tiene influencia sobre las conductas relacionadas con el consumo de tabaco, el uso de drogas y la actividad física y la dieta, así como en la funcionalidad física, la percepción de salud y la salud mental (4-9).

Para comprender la forma como el lugar de residencia impacta en la salud, es necesario hacer algunas precisiones conceptuales. Desde un punto de vista sistémico, el ambiente residencial puede entenderse como producto de una interacción físico-social entre el habitante y su hábitat, la cual tiene lugar en el espacio de la vivienda, a nivel de vecindarios y dentro de una comunidad más amplia (10). El ambiente residencial también puede ser concebido como un sistema proveedor de recursos relacionados con la salud.

Según Bernard y colaboradores, la salud es una expresión de los eventos que acontecen en la vida diaria: necesidades, disponibilidad, acceso y uso de recursos, los cuales se encuentran en distintos dominios (11). El dominio físico natural comprende agua, aire, suelo y clima; el dominio físico construido incluye componentes tales como infraestructura, uso del suelo y conectividad, y el dominio social está dado, en un macronivel, principalmente por la economía y las instituciones, y en un micronivel, por las distintas estructuras comunitarias que tienen representación en el ámbito más local. Las interacciones entre estos dominios, ya sea en términos de proximidad física o por acción de las distintas fuerzas sociales, conformarían las piezas claves para producir el acceso a recursos que pueden beneficiar o deteriorar la salud.

Más aún, la comprensión de estas interrelaciones por parte de los propios residentes de campamentos podría ser relevante para evaluar el impacto positivo o negativo de cualquier intervención en su bienestar. Las comunidades chilenas que viven en campamentos forman núcleos delimitados geográficamente y en situaciones diversas de necesidad $(3,12)$. Estimaciones censales muestran que, comparadas con los promedios nacionales, estas poblaciones son más jóvenes, tienen menos años de escolaridad y mayores tasas de desempleo (12). El alto costo de los terrenos urbanos ha hecho difícil cons- truir viviendas sociales en sectores céntricos y conectados, dando lugar a que las familias permanezcan tiempos prolongados en los campamentos (13). Actualmente, diferentes entidades — entre ellas, asesores inmobiliarios y municipiosparticipan gestionando una vivienda definitiva para estos residentes, interactuando con organizaciones sociales de los campamentos dedicadas no solo a las viviendas sino también a la atención de emergencias y conflictos vecinales (14). Mientras estas organizaciones sociales son consideradas componentes del capital social, capaces de generar beneficios para la comunidad, los problemas sociales en estos barrios - p. ej. vandalismo, consumo de drogas y conflictos entre vecinos- han sido vinculados precisamente a desorganización y estrés social $(5,15,16)$.

Si bien los datos censales constituyen la principal fuente para estudiar la zona de residencia, su utilización presenta algunas limitaciones en casos de comunidades con carencias habitacionales severas. Por un lado, los censos de base poblacional pueden registrar diversas inexactitudes al establecer la ubicación geográfica de estos campamentos, debido a que las comunidades ocupan áreas pequeñas y tienen una disposición territorial heterogénea. Por el otro, se ha señalado que estos censos encuentran dificultades para captar fenómenos sociales que ocurren en el ámbito local, y que dicha carencia podría reducirse mediante el uso de informantes clave (17, 18). Hay asimismo otros enfoques, como es el caso de un diseño transversal, cuya limitación radica en que probablemente no logran una inclusión representativa de esos segmentos de población (19).

En 2007, la organización no gubernamental "Un Techo para Chile" (UTPCH) identificó a la Región Metropolitana (RM) como la principal zona de concentración de asentamientos precarios del país (22,9\%) (3). El catastro nacional de campamentos de UTPCH registró con exactitud los campamentos de todo el país y recolectó datos de informantes clave del campamento sobre las características del lugar de emplazamiento y sus formas de organización, además de un conjunto de problemas sociales percibidos al interior de la comunidad. Dado que hasta el momento no se ha determinado la diversidad de condiciones residenciales en este grupo de población, ni se han propuesto enfoques teóricos para analizar necesidades y oportunidades de investigación en estos grupos, los objetivos del presente estudio fueron identificar dimensiones residenciales que caractericen el ambiente físico y social en los campamentos y construir tipologías que permitan elaborar perfiles con esos atributos residenciales distintivos.

\section{MATERIALES Y MÉTODOS}

Se estudió el universo de campamentos $(n=122)$ de la Región Metropolitana de Chile, los cuales se distribuyeron en 33 de las 52 comunas que componen la Región, con un total de 5599 familias. La construcción del catastro se inició en abril de 2007 a nivel nacional, con la identificación de los campamentos existentes en cada área territorial del país, tomando como fuente los informes de instituciones — públicas y privadas- que trabajan con estas comunidades. Se desarrolló un cuestionario semiestructurado, el cual tuvo una aplicación piloto en miembros de la Corporación Nacional de Dirigentes de Campamentos, para evaluar la pertinencia y aceptabilidad de las preguntas. En el estudio ampliado participaron ocho voluntarios en la RM-estudiantes del área social de UTPCH—, quienes fueron capacitados para aplicar el cuestionario. Fueron entrevistados 122 informantes clave, uno por cada campamento, la mayoría representantes comunitarios electos por votación de la propia comunidad. En caso de ausencia de dirigencia, se entrevistó a aquellas personas con mayor antigüedad en el campamento. La aplicación del cuestionario tuvo una duración aproximada de 20 minutos y sus contenidos surgieron de estudios y experiencias previas que UTPCH había generado desde su formación (20-22). La estructura del cuestionario consideró contenidos generales como región y comuna de procedencia del asentamiento, área urbana (más de 2000 habitantes) o rural, antigüedad de la comunidad (desde la formación del campamento hasta el momento del catastro), propiedad del terreno (estatal, municipal, propia, de terceros o coexistencia de terrenos propios y de uno o más terceros) y número de familias residentes.

En temas más específicos, se incluyeron numerosas variables dicotómicas registradas con el sistema de lista de control (check-list), indagando acerca de la cercanía con respecto a diferentes peligros am- 
bientales (relacionados con vías de mucho tránsito vehicular, canales, riberas de río, quebradas, terrenos con pendiente alta, así como proximidad a desechos industriales, basurales, líneas de tren, torres de alta tensión y plantaciones forestales) y servicios urbanos (puesto de policía, oficina municipal, medios de transporte público, educativos y de salud). La definición de cercanía fue descrita en la metodología del catastro y consistió en distancias al campamento menores a los $500 \mathrm{~m}$ ó $3 \mathrm{~km}$, dependiendo del tipo de servicio, y menores a $3 \mathrm{~km}$ cuando se trató de peligros ambientales (3).

Adicionalmente, se consultó acerca de la existencia de infraestructura comunitaria (cancha deportiva, plaza, parque, sede social e iglesia), organizaciones sociales (juntas de vecinos; comités de allegados, de vivienda y de adelanto; centros juveniles, de madres, de adultos mayores; clubes deportivos y otros), existencia de proyectos de vivienda, con mediación de organismos estatales o privados, y problemas sociales (cesantía laboral, alcoholismo, drogadicción, violencia familiar, narcotráfico, prostitución, discriminación y conflictos entre vecinos o con el municipio).

Otro conjunto de preguntas registró las formas predominantes de acceso a servicios básicos (agua potable, eliminación de excretas y electricidad). Se consideró acceso regular a agua, la conexión por cañería a la red pública e irregular si se obtenía a través de mangueras, tomas a esteros o pozos. La provisión por medio de "camiones-estanque" del municipio fue clasificada como la forma más precaria de acceso al agua. Respecto al sistema de eliminación de excretas, se halló un predominio en el uso de alcantarillado, seguido de fosa séptica (tratamiento primario de aguas negras domésticas) y pozo negro —este último considerado el más precario. Finalmente se preguntó si el modo más común de acceso a electricidad fue a través de la red pública o de otra casa ("colgado"), o si no disponía de este servicio.

En el análisis descriptivo se empleó el área de emplazamiento, la propiedad del terreno y la forma predominante de acceso a servicios básicos. Para el análisis de las otras características, dadas las numerosas variables recolectadas en relación al conjunto de observaciones, se generaron cinco indicadores que resumieron, en escala ordinal, el número de atributos presentes en el lugar. La amenaza ambiental agrupó sus peligros en la proximidad, entendiendo que los campamentos que concentran más peligros tienen una mayor probabilidad de ocurrencia de accidentes o de eventos desastrosos (23). Asimismo, se recurrió a la categorización de servicios y de infraestructura comunitaria para definir su grado de disponibilidad, descontando que una menor concentración de estos servicios genera condiciones más deficitarias. De acuerdo a la revisión teórica, se empleó un indicador de diversidad de organizaciones comunitarias como medida indirecta de la capacidad de acción colectiva en los campamentos. Aquellos que presentaron mayor diversidad agruparon un mayor número de organizaciones sociales. Por último, los problemas sociales fueron consolidados bajo el término "estrés social" para caracterizar a los campamentos que agruparon un mayor número de estos problemas percibidos.

Se utilizaron dos técnicas multivariadas complementarias para disminuir la dimensionalidad del número de variables e identificar las tipologías residenciales que caracterizaban el universo de campamentos. La primera técnica fue el análisis factorial exploratorio (AFE), empleado cuando no se conocen las relaciones entre las variables o cuando se desea reducir el volumen de información de las variables originales identificando factores comunes entre ellas. A partir de este proceso se obtienen nuevas dimensiones que son una combinación lineal de variables y que representan la estructura subyacente (o latente) de las categorías sobre las cuales se agrupan las variables originales (24).

Los factores son generados a partir de una matriz de correlaciones. En este caso, las variables empleadas fueron dicotómicas u ordinales, de modo que se empleó un AFE sobre una matriz de correlación tetracórica o policórica, apropiada a dicho tipo de escala de medida (25). En cuanto al procedimiento para extraer los factores, se decidió usar la extracción por componentes principales debido a que es una solución eficiente cuando el objetivo es conseguir la mayor información posible proveniente de la asociación entre las variables y su factor común (26).

El paso siguiente consistió en mejorar la interpretación de la solución que provee el análisis empleando un método de rotación en un espacio factorial. En este sentido, se optó por la rotación oblicua Promax, en particular porque supone que los factores están correlacionados, lo cual era esperable por la naturaleza de las variables involucradas (26). Si bien no existe un criterio absoluto para determinar el número de factores a retener, se utilizaron conjuntamente los dos criterios más aceptados: el criterio de Kaiser, que especifica la retención de factores cuyos valores propios de la matriz de correlaciones sean mayor a uno, y el gráfico de screeplot $(25,27)$. Para la interpretación de los factores, en cada dimensión se extrajeron las variables que tuvieron cargas factoriales mayores a 0,5. Posteriormente se efectuó el cálculo de puntuaciones factoriales para cada observación, el cual permitió asignar a cada campamento una puntuación basada en la existencia y combinación de características del lugar.

La segunda técnica multivariada que se empleó fue el análisis de agrupamientos (AG), con la cual se busca reunir y clasificar los elementos de una muestra o población en subgrupos sobre la base de una medida de similaridad $(28,29)$. En estudios diversos, la aplicación de esta técnica - posterior a un análisis factorial- ha reportado beneficios importantes en la interpretación de los resultados (30-33). Los autores destacan que el AFE por sí solo, aun cuando permite extraer las principales dimensiones de un modelo de estudio, es limitado en mostrar las combinaciones de las dimensiones resultantes, y que el AG fortalece el análisis al establecer relaciones entre las dimensiones generadas por el análisis factorial. Y recíprocamente, el empleo del AG como método único no permite discriminar las variables que no contribuyen al constructo teórico, una función que sí cumple el AFE.

El análisis de tipologías agrupó y discriminó grupos sobre la base de una medida de distancia o similaridad. Se emplearon dos técnicas de agrupamiento que, combinadas, generaron beneficios en la identificación de grupos: primero una jerárquica aglomerativa y, luego, una no jerárquica de k-medias sobre las puntuaciones factoriales (29). La técnica jerárquica permitió establecer el número de grupos iniciales y su perfil central. En este estudio la extracción de los primeros agrupamientos de cada factor se realizó con el método de Ward, basado en la suma de cuadrados de la distancia euclidiana. Posteriormente, y considerando el número de grupos formados en la etapa inicial, se utilizó la técnica de k-medias para obtener la solución más adecuada (29). 
Para determinar el número de grupos iniciales, se evaluó el cambio en el coeficiente de aglomeración en cada etapa del proceso jerárquico. Según las reglas de aglomeración, los coeficientes pequeños muestran homogeneidad al interior de los grupos, mientras que un gran incremento en el coeficiente indica un cambio y la formación de un nuevo grupo (28). Una vez logrados los agrupamientos finales con la técnica de k-medias, se compararon los resultados numéricos obtenidos con la técnica aglomerativa y la de k-medias para identificar el patrón de agrupamiento. Luego se evaluó la estabilidad de las soluciones mediante un segundo análisis alternativo no jerárquico utilizando k-medias, pero ahora aleatorizando los puntos de inicio (29). Finalmente se analizó el perfil de los agrupamientos a través de la distribución de las variables originales en cada grupo.

En el análisis de la antigüedad y del número de familias se incluyeron la mediana y valores mínimos y máximos. Se empleó un diagrama para representar las características de cada dimensión y la posición jerárquica de cada agrupamiento en torno a la variable-componente. Dicha jerarquía fue definida por el cálculo de una razón que proviene de dividir la proporción del atributo en cada agrupamiento y la proporción del atributo en el universo de campamentos. En el diagrama, los óvalos representan dicha jerarquía, es decir, la intensidad con que un atributo estuvo presente. Un óvalo más grande indica que el atributo de interés se concentró más en un determinado grupo (porcentaje-atributogrupo/porcentaje-atributo-universo $>1$ ), mientras que uno más pequeño, que el atributo estuvo menos presente (porcentaje-atributo-grupo/porcentajeatributo-universo $<1$ ). Dependiendo de la característica observada, se interpretó como déficit cuando una característica deseable se presentó en menor proporción en un determinado grupo.

\section{RESULTADOS}

La mayoría de los campamentos estaban localizados en sectores urbanos y en terrenos de propiedad estatal. En el universo de campamentos, el déficit predominante se dio en los servicios básicos, mientras que en infraestructura y otros servicios urbanos no se observaron categorías de déficit predominantes. La tendencia en el conjunto de campamentos

CUADRO 1. Características residenciales predominantes en campamentos de la Región Metropolitana de Chile, 2007

\begin{tabular}{|c|c|c|}
\hline \multirow[b]{2}{*}{ Característica } & \multicolumn{2}{|c|}{$n=122$} \\
\hline & No. & $\%$ \\
\hline Localización en área urbana & 87 & 71,3 \\
\hline \multicolumn{3}{|l|}{ Propiedad del terreno } \\
\hline Estatal & 54 & 44,3 \\
\hline Privado & 47 & 38,5 \\
\hline Mixto (privado/propios dueños) ${ }^{\mathrm{a}}$ & 10 & 8,2 \\
\hline No sabe & 11 & 9,0 \\
\hline \multicolumn{3}{|l|}{ Acceso a agua potable $(\mathrm{fp})^{b}$} \\
\hline Red pública directa & 37 & 30,3 \\
\hline Irregular (mangueras, toma de esteros) & 61 & 50,0 \\
\hline Camión-estanque & 24 & 19,7 \\
\hline \multicolumn{3}{|l|}{ Eliminación de excretas $(\mathrm{fp})^{\mathrm{b}}$} \\
\hline Red pública/fosa sépticac & 34 & 27,9 \\
\hline Pozo negro/depósito (sin tratamiento) & 88 & 72,1 \\
\hline \multicolumn{3}{|l|}{ Acceso a electricidad $(f p)^{b}$} \\
\hline Red pública & 57 & 46,7 \\
\hline Irregular (a través de otra casa,"colgado") & 65 & 53,3 \\
\hline \multicolumn{3}{|l|}{ Nivel de amenaza ambiental } \\
\hline Bajo: $\leq 1$ peligro ambiental & 40 & 32,8 \\
\hline Medio: $2-4$ peligros & 52 & 42,6 \\
\hline Alto: $\geq 5$ peligros & 30 & 24,6 \\
\hline \multicolumn{3}{|l|}{ Disponibilidad de servicios urbanos } \\
\hline Bajo: ningún servicio & 38 & 31,1 \\
\hline Medio: $2-4$ servicios & 50 & 41,0 \\
\hline Alto: todos los servicios & 34 & 27,9 \\
\hline \multicolumn{3}{|l|}{ Disponibilidad de infraestructura comunitaria } \\
\hline Bajo: $\leq 1$ tipo & 47 & 38,5 \\
\hline Medio: 2 ó 3 tipos & 28 & 23,0 \\
\hline Alto: todos los tipos & 47 & 38,5 \\
\hline \multicolumn{3}{|l|}{ Diversidad de organizaciones sociales } \\
\hline Bajo: 1 forma de organización & 71 & 58,1 \\
\hline Medio: 2 formas & 23 & 18,9 \\
\hline Alto: $\geq 3$ formas & 28 & 23,0 \\
\hline Existencia de proyecto habitacional & 59 & 48,4 \\
\hline \multicolumn{3}{|l|}{ Nivel de estrés social } \\
\hline Bajo: $\leq 1$ problema social percibido & 32 & 26,3 \\
\hline Medio: 2-4 problemas & 37 & 30,3 \\
\hline Alto: $\geq 5$ problemas & 53 & 43,4 \\
\hline
\end{tabular}

Fuente: elaboración de los autores con datos del Catastro Nacional de Campamentos, realizado por la fundación Un Techo para Chile, 2007.

a Terrenos que son propiedad tanto de residentes del campamento como de terceros.

${ }^{b}$ fp: forma predominante.

c Se generó una sola categoría (Red pública/fosa séptica) porque ambas formas implican un manejo más apropiado de las excretas que el pozo negro. Además, se registró solo un campamento con acceso predominante a alcantarillado.

fue hacia la formación de organizaciones únicas y la notificación de un mayor número de problemas en la comunidad (cuadro 1). Se obtuvieron tres factores que explicaban 51,1\% de la varianza y cuyas correlaciones oscilaron entre $0,21 \mathrm{y}$ 0,88 . El primer conjunto de variables, definidas como "respuesta social local", reflejaba a la comunidad residente y su acción colectiva (cuadro 2). Los mayores puntajes correspondieron a los campamentos que disponían de proyectos habitacionales, con mayor número de equipamientos comunitarios y una mayor diversidad de organizaciones sociales. Parte de este factor fue la variable estrés social, que resultó correlacionada con las características previas. En el segundo conjunto de variables, que describieron el "ambiente extracomunitario", los puntajes más altos correspondieron a los campamentos emplazados en áreas urbanas, con mayor diversidad de servicios y menor amenaza ambiental en la vecindad. Finalmente, el tercer factor fue formado por variables que respondieron a la "disponibilidad de servicios básicos", cuyos puntajes más altos fueron a los asentamientos donde predominó la conexión directa a red eléctrica y el acceso a agua potable por cañería.

En el análisis de agrupamiento mediante el empleo de las cargas factoriales, una solución de cuatro grupos mostró mayor estabilidad en la clasificación de los asentamientos. A continuación se 
CUADRO 2. Puntajes obtenidos en análisis factorial de características residenciales en campamentos de la Región Metropolitana de Chile, $2007(n=122)$

\begin{tabular}{lccc}
\hline \multicolumn{1}{c}{ Característica } & $\begin{array}{c}\text { Respuesta } \\
\text { social } \\
\text { local }\end{array}$ & $\begin{array}{c}\text { Ambiente } \\
\text { extra- } \\
\text { comunitario }\end{array}$ & $\begin{array}{c}\text { Disponibilidad } \\
\text { de servicios } \\
\text { básicos }\end{array}$ \\
\hline Área urbana & 0,0348 & $0,8973^{\mathrm{a}}$ & $-0,1052$ \\
Propiedad del terreno estatal & $-0,0772$ & $-0,1125$ & $-0,0263$ \\
Eliminación de excretas (fp) & 0,2755 & 0,0771 & 0,3652 \\
Acceso a agua potable (fp) & 0,1460 & 0,1720 & $0,7498^{\mathrm{a}}$ \\
Acceso a electricidad (fp) & $-0,0922$ & $-0,3261$ & $0,8208^{\mathrm{a}}$ \\
Nivel de amenaza ambiental & 0,4580 & $-0,5198^{\mathrm{a}}$ & $-0,3769$ \\
Disponibilidad de servicios urbanos & 0,0971 & $0,6065^{\mathrm{a}}$ & $-0,0023$ \\
Disponibilidad de infraestructura comunitaria & $0,7148^{\mathrm{a}}$ & 0,0460 & 0,0978 \\
Diversidad de organizaciones sociales & $0,6432^{\mathrm{a}}$ & $-0,4541$ & 0,2412 \\
Existencia de proyecto habitacional & $0,7565^{\mathrm{a}}$ & 0,1641 & $-0,1383$ \\
Nivel de estrés social & $0,6087^{\mathrm{a}}$ & 0,0343 & $-0,0114$
\end{tabular}

Fuente: elaboración de los autores con datos del Catastro Nacional de Campamentos, realizado por la fundación Un Techo para Chile, 2007.

a Variables que más contribuyen a los respectivos factores.

b fp: forma predominante.

presenta una síntesis de los perfiles resultantes (cuadro 3):

Perfil urbano con baja precariedad de servicios básicos (Perfil 1 = 30 campamentos). Reúne campamentos con una mediana de tamaño y de antigüedad de 33 familias y 14 años respectivamente, donde predomina el acceso regular a energía eléctrica y agua potable de red. El entorno representa una baja amenaza ambiental para la comunidad, en tanto que la disponibilidad de servicios es media y no constituye una característica distintiva. Las comunidades de este grupo no concentran en forma destacada proyectos habitacionales, pero disponen de infraestructura comunitaria y variadas organizaciones comunitarias. Este grupo concentra un alto estrés social.

Perfil urbano-rural con alto potencial de respuesta social local (Perfil $2=32$ campamentos). Reúne campamentos con una mediana de tamaño y de antigüedad de 44 familias y 13 años respectivamente, y predominan los sistemas de saneamiento irregulares, incluyendo comunidades que reciben agua de camionesestanque municipales. La disponibilidad de servicios urbanos también es media y presenta la más baja amenaza ambiental. Un rasgo distintivo de este grupo es la respuesta social local, la cual reúne notoriamente a las comunidades con proyectos de vivienda, mayor diversidad de organizaciones y más equipamiento comunitario. Estas mismas comunidades son las que concentran el mayor número de problemas sociales percibidos.

Perfil urbano con alta amenaza ambiental (Perfil 3 = 43 campamentos). Constituye el grupo mayoritario y reúne un importante número de micro-campamentos ( $<20$ familias) con una antigüedad mediana de 15 años. En este grupo se registra la mayor irregularidad en el acceso a electricidad y agua de todos los grupos. En el entorno, la disponibilidad de servicios fue media y similar a la de los perfiles urbanos restantes, aunque reúne el mayor número de peligros ambientales. La respuesta local es pobre en consideración a que la mitad de los asentamientos no participan de proyectos de vivienda,

CUADRO 3. Composición de los perfiles residenciales, en relación a variables seleccionadas, en campamentos de la Región Metropolitana de Chile, 2007

\begin{tabular}{|c|c|c|c|c|c|c|}
\hline \multirow[b]{2}{*}{ Características residenciales } & \multirow[b]{2}{*}{ Categoría } & \multicolumn{5}{|c|}{ Porcentaje } \\
\hline & & $\begin{array}{l}\text { Perfil } 1 \\
(n=30)\end{array}$ & $\begin{array}{l}\text { Perfil } 2 \\
(n=32)\end{array}$ & $\begin{array}{l}\text { Perfil } 3 \\
(n=43)\end{array}$ & $\begin{array}{l}\text { Perfil } 4 \\
(n=17)\end{array}$ & $\begin{array}{c}\text { Total } \\
(n=122)\end{array}$ \\
\hline Área & Urbana & 76,7 & 59,4 & 93,0 & 29,4 & 71,3 \\
\hline Acceso a electricidad (fp) & Irregular & 3,3 & 62,5 & 81,4 & 52,9 & 53,3 \\
\hline \multirow[t]{3}{*}{ Acceso a agua potable (fp) } & Camiones & 0,0 & 25,0 & 16,3 & 52,9 & 19,7 \\
\hline & Irregular & 6,7 & 59,4 & 74,4 & 47,1 & 50,0 \\
\hline & Regular & 93,3 & 15,6 & 9,3 & 0,0 & 30,3 \\
\hline \multirow[t]{3}{*}{ Nivel de amenaza ambiental } & Alto & 3,4 & 3,1 & 48,8 & 23,5 & 24,6 \\
\hline & Medio & 43,3 & 31,3 & 41,9 & 64,7 & 42,6 \\
\hline & Bajo & 53,3 & 65,6 & 9,3 & 11,8 & 32,8 \\
\hline \multirow[t]{3}{*}{ Nivel de servicios urbanos } & Bajo & 23,3 & 31,3 & 18,6 & 76,5 & 31,2 \\
\hline & Medio & 46,7 & 43,7 & 44,2 & 17,7 & 40,9 \\
\hline & Alto & 30,0 & 25,0 & 37,2 & 5,8 & 27,9 \\
\hline \multirow[t]{3}{*}{ Nivel de infraestructura comunitaria } & Bajo & 20,0 & 6,2 & 53,5 & 94,1 & 38,5 \\
\hline & Medio & 36,7 & 18,8 & 25,6 & 0,0 & 23,0 \\
\hline & Alto & 43,3 & 75,0 & 20,9 & 5,9 & 38,5 \\
\hline \multirow[t]{3}{*}{ Diversidad de organizaciones sociales } & Bajo & 46,7 & 31,2 & 79,1 & 76,4 & 58,2 \\
\hline & Medio & 20,0 & 21,9 & 18,6 & 11,8 & 18,9 \\
\hline & Alto & 33,3 & 46,9 & 2,3 & 11,8 & 22,9 \\
\hline Existencia de proyecto habitacional & No & 53,3 & 21,9 & 55,8 & 94,1 & 51,6 \\
\hline \multirow[t]{3}{*}{ Nivel de estrés social } & Alto & 46,7 & 68,7 & 37,2 & 5,9 & 43,4 \\
\hline & Medio & 23,3 & 31,3 & 30,2 & 41,2 & 30,3 \\
\hline & Bajo & 30,0 & 0,0 & 32,6 & 52,9 & 26,3 \\
\hline Tamaño: mediana (mín-máx) & No. familias & $33(8-360)$ & $44(9-320)$ & $20(8-120)$ & $13(8-42)$ & $28(8-360)$ \\
\hline Antigüedad: mediana (mín-máx) & Años & $14(2-82)$ & $13(2-87)$ & $15(1-50)$ & $18(2-57)$ & $14(1-87)$ \\
\hline
\end{tabular}

Fuente: elaboración de los autores con datos del Catastro Nacional de Campamentos, realizado por la fundación Un Techo para Chile, 2007.

Nota: Perfil 1, urbano con baja precariedad de servicios básicos; Perfil 2, urbano-rural con alto potencial de respuesta social local; Perfil 3, urbano con alta amenaza ambiental; Perfil 4, rural con bajo potencial de respuesta social local. Fp: forma predominante. 
FIGURA 1. Jerarquía de perfiles residenciales en campamentos de la Región Metropolitana de Chile, 2007

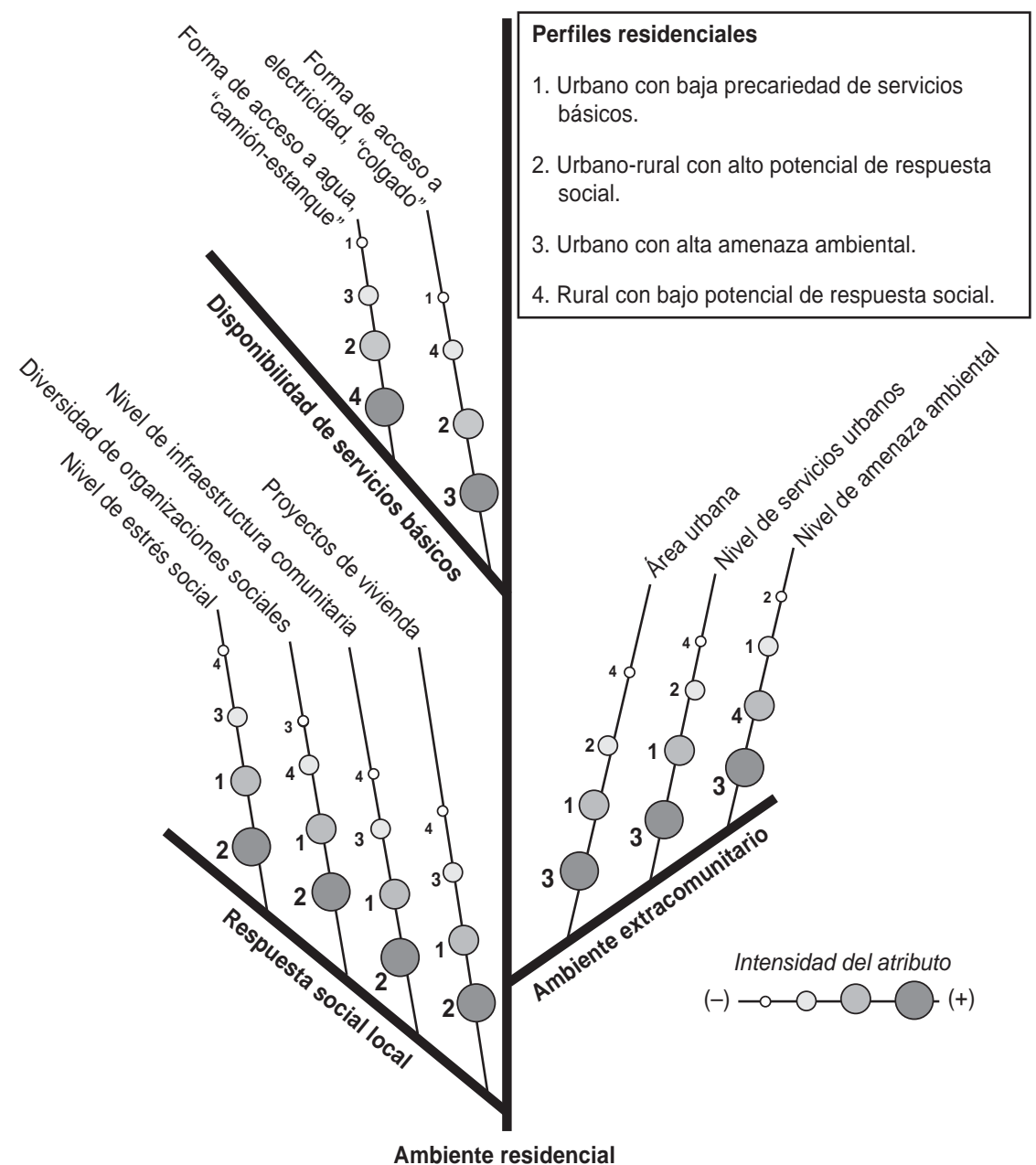

Fuente: elaboración de los autores con datos del Catastro Nacional de Campamentos, realizado por la fundación Un Techo para Chile, 2007.

existe una importante carencia de equipamiento comunitario y presentan la más baja diversidad en organizaciones. La concentración de problemas sociales fue baja.

Perfil rural con bajo potencial de respuesta social local (Perfil $4=17$ campamentos). Reúne los micro-campamentos con menor número de familias $(\leq 13)$, y con una antigüedad mediana de 18 años. Predomina el acceso irregular a servicios de electricidad y registra el máximo déficit en acceso a agua potable, de la cual se abastece por medio de camionesestanque municipales. Otro rasgo distintivo es que sus asentamientos tienen la mayor carencia de servicios urbanos, no participan en proyectos de vivienda, no disponen de equipamiento comunitario y registran menor diversidad de organizaciones. Es el grupo que registra menor número de estresores sociales.
En la figura 1 se pueden observar tres ejes que representan las tres dimensiones residenciales formadas: respuesta social local, ambiente extracomunitario y disponibilidad de servicios básicos. En función de esos ejes, los perfiles tres (urbano) y cuatro (rural) lideran el déficit de servicios básicos y concentran mayor amenaza ambiental. Asimismo, se ubican en las peores posiciones en cuanto a acción colectiva y, por el contrario, en la mejor posición respecto a estrés social. Los perfiles residenciales restantes disponen en general de un mejor contexto de servicios, infraestructura y organización, pero a diferencia de los perfiles previos, concentran más estresores sociales.

\section{DISCUSIÓN}

Por medio de análisis factorial, se retuvieron tres dimensiones que diferencian tres perfiles residenciales, uno centrado en la respuesta potencial de la comunidad frente a necesidades y dos que describen el área de servicios: básicos (agua y electricidad) y urbanos de integración a las áreas geográficas (transporte, salud, educación, seguridad). El componente principal de la dimensión respuesta social local fue la existencia de proyectos habitacionales, variable que alcanzó una alta correlación con la infraestructura, el número de organizaciones y los problemas percibidos en la comunidad. Este resultado concuerda con estudios realizados en asentamientos chilenos donde, en la percepción de pobladores y dirigentes, la vivienda se describía como una meta central, siendo las organizaciones sociales funcionales a ese objetivo (14). También fue coherente la relación de estas variables con el número de equipamientos comunitarios, concordando con investigadores que hallaron un vínculo entre la infraestructura urbana (p. ej. áreas verdes y lugares para practicar deportes) y el grado de participación individual, toda vez que estos lugares implican oportunidades de encuentro entre personas y son facilitadores de la interacción social (34). Estos aspectos en conjunto pueden ser considerados atributos beneficiosos para la comunidad, pues representan movilización hacia objetivos comunes y evidencian presencia de redes y oportunidades de encuentro entre vecinos. Sin embargo, las variables de esta dimensión también mostraron una correlación directa con el número de problemas sociales percibidos, lo cual podría contrastar con estudios que observan una relación inversa entre medidas de capital social y eventos negativos de la vida (p. ej. pérdida de trabajo, inseguridad, adicciones y pérdida de salud mental), en el entendido de que una comunidad con mayores y mejores redes promueve el bienestar individual. Si bien a partir de estos resultados no se puede comprender el origen de estas relaciones, una posible respuesta podría estar en el número de familias que agrupa cada campamento $(15,16,35)$. En efecto, los grupos con mayor déficit en proyectos y formas de organización incluyeron a los micro-campamentos (grupos 3 y 4). Las mayores dificultades que tienen esos grupos pueden ser explicadas por la necesidad obligada de crear alianzas con otras comunidades para postular a la vivienda. Adicionalmente, los grupos de familias tienen vínculos sanguíneos y sus dirigentes pueden sentirse 
menos proclives a explicitar los problemas sociales que los aquejan. Consistente con esto, en una comunidad mayor las dificultades sociales cruzan la barrera personal en la medida que las familias con mayores carencias en el hogar, o con determinadas conductas sociales, son consideradas como un problema para otros vecinos.

Para algunos autores la presencia de altos índices de problemas sociales $y$, a la vez, de participación social no debe extrañar debido a que la existencia de problemas también puede generar un nivel de tensión y movilización que impulse la asociatividad en una comunidad (36). Además se ha descrito que, a nivel comunitario, se producen interacciones sociales que brindan determinados niveles de apoyo social y que pueden traducirse en recursos (p. ej. materiales, ayuda, pertenencia e integración) que amortiguan situaciones de estrés para la comunidad (37). En este sentido los resultados del presente estudio, aun cuando no permiten establecer la relación temporal entre las variables, sí posibilitan plantear algunas hipótesis en ciertos grupos, especialmente para los campamentos que disponen de diversas organizaciones y movilización hacia proyectos de vivienda y que son, coincidentemente, los que acusan mayor concentración de problemas sociales.

El área de residencia fue el componente principal para el ambiente extracomunitario, correlacionando directamente con la disponibilidad de servicios urbanos e inversamente con el grado de amenaza ambiental, y sugiriendo así que los campamentos emplazados en áreas urbanas disponen de condiciones de habitabilidad más favorables que los perfiles que concentraron mayor número de campamentos rurales. Aun así, la situación de disponibilidad de servicios fue relativamente homogénea entre los grupos, lo que es probable si se toma en consideración que una necesidad en los residentes de campamento es radicarse en un lugar bien abastecido.

El análisis de agrupamientos, a diferencia del análisis factorial, reveló la existencia de cuatro tipologías en la RM. Dos perfiles ( 1 y 3 ) reunieron campamentos emplazados en su totalidad en el área urbana. No obstante, mientras que el Perfil 1 tendió a concentrar los asentamientos con mejor acceso a servicios básicos de agua y electricidad, el Perfil 3 no sólo registró carencias de estos servicios, sino que destacó por el número de peligros ambientales próximos al lugar de emplazamiento. Si bien la tipología de dichos peligros no fue analizada, su concentración en determinados lugares indica que hay comunidades con mayor vulnerabilidad ambiental, y que esto puede incidir en un mayor riesgo de accidentes y de exposición directa a contaminantes.

Los grupos restantes concentran un mayor número de asentamientos rurales y, dentro de ellos, el Perfil 4 sobresale como el más crítico en cuanto a su situación residencial porque aglutinó las peores condiciones de habitabilidad: aislamiento territorial, amenaza ambiental y precariedad de servicios. Sin embargo, en estos grupos se observó un menor número de problemas sociales que en los asentamientos urbanos, inclusive inferior a los micro-campamentos de naturaleza urbana. Tal como ya se planteó, el menor número de familias en estos grupos podría haber incidido en la menor notificación de problemas y, a la vez, en una inferior capacidad de respuesta frente a las necesidades. También se ha descrito un menor estrés social en áreas rurales donde, pese a padecer un relativo aislamiento territorial en comparación con zonas urbanas, predominan actividades de auto sustento menos tensionantes que las que debe emprender la población pobre urbana, obligada a integrarse a un mercado laboral (38).

La antigüedad de los campamentos ha sido mencionada en la literatura como un determinante en la conformación de este tipo de asentamientos, pues es un factor que incide en las oportunidades de acceso a servicios básicos y en la estabilidad del colectivo $(1,19)$. En este trabajo los perfiles residenciales mostraron cierta homogeneidad en cuanto al tiempo transcurrido desde su formación, a diferencia de la variable de tamaño, que mostró diferencias según tipologías.

Los resultados del presente estudio deben ser interpretados con cautela, en razón de que los datos empleados pertenecen a un catastro cuya finalidad es establecer un marco general de prioridades de intervención, sin considerar casos individuales ni otros procesos sociales (p. ej. capital social o constructos relacionados) que pudieran caracterizar mejor el dominio social de estas comunidades. De todos modos, los indicadores obtenidos provienen de todo el conjunto de campamentos de la Región Metropolitana, los cuales han sido identificados te- rritorialmente con gran exactitud por la institución responsable del catastro.

El empleo de informantes clave, por otro lado, constituyó un aporte en la captura de características de la comunidad. $\mathrm{Al}$ respecto, se ha señalado que los residentes sin un rol específico en la comunidad suelen no estar bien enterados de lo que acontece en sus barrios y, por lo tanto, la información que proporcionan podría introducir error y sesgo en las estimaciones $(18,39)$. En este caso, el catastro fue aplicado por personas familiarizadas con la dinámica de los campamentos y los informantes mostraron disposición a dar respuestas que no serían confiables en otro contexto de relaciones.

\section{Conclusiones}

Los resultados obtenidos en el presente trabajo dan cuenta de tres grandes dimensiones residenciales que caracterizan a los campamentos de la Región Metropolitana de Chile: una dimensión social (respuesta social local), otra relativa a la disponibilidad de servicios básicos y una tercera de contexto, que agrupa las condiciones externas al campamento (ambiente extracomunitario). La identificación de estas dimensiones concuerda con los modelos teóricos revisados en cuanto a que el ambiente residencial posee atributos que están en el dominio físico representados, en este caso, por los servicios dentro y fuera del campamento, así como en el dominio social donde se manifiesta la comunidad.

La dimensión más determinante en el modelo fue la respuesta social local, dado que explica por sí sola la mayor varianza en la correlación de sus variables, seguida por el ambiente extracomunitario $\mathrm{y}$, por último, la disponibilidad de servicios básicos. La variable más representativa de la dimensión social fue la existencia de proyectos de vivienda, seguida por el nivel de infraestructura comunitaria. El área urbana fue determinante en la formación de la dimensión extracomunitaria, mientras que para la dimensión de servicios básicos lo fue la variable de acceso a electricidad regular. La presencia de estas variables como determinantes del perfil de sus dimensiones sugiere que pueden ser indicadores sociales relevantes para el seguimiento de estas comunidades en un nuevo catastro.

Se identificaron a su vez cuatro perfiles residenciales que muestran heterogeneidad en los campamentos de la Región 
Metropolitana. Las tipologías sugieren que los grupos que se encuentran mejor posicionados en algún ámbito residencial (p. ej. más servicios urbanos) no necesariamente están protegidos respecto de otras características del medio (p. ej. los peligros ambientales o el estrés social). En particular, en esos campamentos se genera una compleja relación de factores que tienden al déficit, a la presencia de amenazas y a la ausencia de proyectos de vivienda - aunque incluso así dan cuenta de un menor estrés social. Asimismo, la condición de microcampamento surgió como un potencial factor involucrado, pero esta hipótesis requiere ser estudiada con mayor profundidad. Las tipologías generadas advierten la necesidad de considerar, en el marco de cualquier intervención -habitacional, social o específica de la salud-, la presencia de variables residenciales de distintos dominios, que pueden o no estar interrelacionadas y consecuentemente incidir tanto en el diseño como en la interpretación de los resultados de una acción determinada.

Como ya se ha planteado en este artículo, el estudio transversal que entrega el Catastro Nacional de Campamentos, realizado por la fundación Un Techo para Chile en 2007, impide observar la continuidad o discontinuidad de indicadores en la comunidad. De allí que no sea posible probar que la existencia de un determinado número de recursos físi- cos en el entorno próximo, de proyectos de vivienda $u$ organizaciones sociales, se traduzca necesariamente en una comunidad sustentable o saludable que provea beneficios en el largo plazo para sus residentes. Si se ha de lograr tal objetivo, será necesario estudiar de modo longitudinal los campamentos, los componentes del dominio social y su interacción con el medio físico. Hará falta especialmente profundizar en la naturaleza de las organizaciones sociales, las capacidades instaladas, las trayectorias de participación o los tipos de liderazgo, como una manera de determinar en qué medida las organizaciones sociales son persistentes y pueden llegar a intervenir en otros ámbitos del bienestar comunitario.

Asimismo será preciso cuantificar la participación de todos los habitantes (a nivel individual o de hogares) para detectar los grados de compromiso y las capacidades disponibles para enfrentar efectivamente las necesidades y los problemas sociales existentes. Estos son pasos necesarios en la lucha contra ciertos problemas percibidos por la comunidad (p. ej. el consumo de drogas o deterioro del ambiente), donde una acción coordinada de la comunidad con las redes sociales puede significar una fuente de apoyo social. La razón por la que esos aspectos revisten particular importancia es debido a que las familias de los campamentos están en un proceso de intervención habitacional con el apoyo de distintos actores que aportan recursos sociales y monetarios, y resultaría relevante examinar con mayor detención la magnitud y la continuidad del apoyo externo que reciben. Si esta asistencia se dirige solamente a la obtención de la vivienda podría resultar poco efectiva para movilizar otro tipo de actitudes y comportamientos que tengan impactos más duraderos en el bienestar.

Finalmente, resulta clara la necesidad de un análisis que permita ampliar y optimizar la identificación de perfiles residenciales de estas comunidades a nivel nacional, particularmente a través de técnicas basadas en el análisis geográfico. Efectivamente, la metodología utilizada en este estudio de datos secundarios puede ser replicada empleando datos nacionales para observar la formación de tipologías que reflejen no solo mayores o menores niveles de déficit, sino nuevas necesidades que podrán ser abordadas desde los diferentes sectores responsables de incrementar la calidad de vida en las comunidades más pobres.

Agradecimientos. Este trabajo se realizó bajo el auspicio de International Training and Research in Environmental and Occupational Health, Fogarty International Center (Atlanta, USA) (PROYECTO FOGARTY No D43TW005746) en colaboración con la Escuela de Salud Pública, Facultad de Medicina, Universidad de Chile.

\section{REFERENCIAS}

1. Winchester L. El desarrollo sostenible de los asentamientos humanos en América Latina y el Caribe. Publicación No 99, serie Medio Ambiente y Desarrollo. Santiago de Chile: Comisión Económica para América Latina y el Caribe; 2006.

2. Mac Donald J. Pobreza y precariedad del hábitat en ciudades de América Latina y el Caribe. Publicación $\mathrm{N}^{\circ}$ 38; LC/L.2214-P. Santiago de Chile: Comisión Económica para América Latina y el Caribe; 2004.

3. Centro de Investigación Social (CIS), Un Techo para Chile (UTPCH). Catastro Nacional de Campamentos. Santiago de Chile: CIS/UPCH; 2007.

4. Yen IH, Syme SL. The social environment and health: a discussion of the epidemiologic literature. Annu Rev Public Health. 1999;20:287-308.

5. Steptoe A, Feldman PJ. Neighborhood problems as sources of chronic stress: development of a measure of neighborhood problems, and associations with socioeconomic status and health. Ann Behav Med. 2001;23(3):177-85.
6. Latkin CA, Curry AD. Stressful neighborhoods and depression: a prospective study of the impact of neighborhood disorder. J Health Soc Behav. 2003;44(1):34-44.

7. Poortinga W. Perceptions of the environment, physical activity, and obesity. Soc Sci Med. 2006;63(11):2835-46.

8. Winkler E, Turrell G, Patterson C. Does living in a disadvantaged area mean fewer opportunities to purchase fresh fruit and vegetables in the area? Findings from the Brisbane Food Study. Health Place. 2006;12:306-19.

9. Ellaway A, Kirk A, Macintyre S, Mutrie N. Nowhere to play? The relationship between the location of outdoor play areas and deprivation in Glasgow. Health Place. 2007;13:557-61.

10. Toro A, Jirón P, Goldsack L. Análisis e incorporación de factores de calidad habitacional en el diseño de las viviendas sociales en Chile. Propuesta metodológica para un enfoque integral de la calidad residencial. En: Sepúlveda O, ed. Calidad del hábitat residencial. Santiago de Chile: Universidad de Chile; 2003. P. 149.
11. Bernard $P$, Charafeddine R, Frohlich KL, Daniel M, Kestens Y, Potvin L. Health inequalities and place: a theoretical conception of neighbourhood. Soc Sci Med. 2007;65(9):1839-52.

12. Chile, Ministerio de Vivienda y Urbanismo, Universidad de Chile. Catastro de Campamentos y Asentamientos Irregulares, Chile. Publicación técnica s/n; 11 vol. Santiago de Chile: Ministerio de Vivienda y Urbanismo/ Universidad de Chile; 1997.

13. Atria J. Capital social y voluntariado: claves para un financiamiento solidario de la vivienda social. El caso de la fundación Un Techo para Chile. Revista INVI. 2007;22(61):13-30.

14. Campo A, Guerrero N, Machuca D, Rebolledo L. Prácticas políticas en campamentos y barrios: algunas recomendaciones para su fortalecimiento. Documento de Trabajo No. 2. Santiago de Chile: Un Techo Para Chile; 2009. P. 14.

15. Kripper C, Sapag J. Capital social y salud en América Latina y el Caribe: una revisión sistemática. Rev Panam Salud Publica. 2009;25(2): $162-70$. 
16. Echeverria S, Diez-Roux AV, Shea S, Borrell LN, Jackson S. Associations of neighborhood problems and neighborhood social cohesion with mental health and health behaviors: the Multi-Ethnic Study of Atherosclerosis. Health Place. 2008;14(4):853-65.

17. Houston MJ, Sudman S. A methodological assessment of the use of key informants. Soc Sci Res. 1975;4(2):151-64.

18. Pauwels L, Hardyns W. Measuring Community (Dis) Organizational Processes through Key Informant Analysis. European Journal of Criminology. 2009;6(5):401-17.

19. Centro Latinoamericano de Demografía (CELADE). Población y vivienda en asentamientos precarios. Publicación $\mathrm{N}^{\circ} 299 ; 7$ vol. Santiago de Chile: Ministerio de Vivienda y Urbanismo; 1998

20. Alvarado M, Gallegos S. El mercado laboral en campamentos. Santiago de Chile: Un Techo para Chile/Universidad de Chile; 2004.

21. Bedregal P, Zavala C, Atria J, Nuñez G. Calidad de vida y necesidades de salud en campamentos: una mirada fundamental a la extrema pobreza. Revista CIS (Centro de Investigación Social, UTPCH). 2006;5(8):2-9.

22. Mardones N. Dinámica de la pobreza. Santiago de Chile: Un Techo para Chile/Escuela de Ingeniería Comercial Universidad de Los Andes; 2007.

23. United Nations Disaster Relief Organization (UNDRO). Classification of concepts and terms. Natural disasters and vulnerability analisys. Geneva: Office of the United Nations Relief Disasters Coordinator; 1979. P. 49.
24. Vivanco M. Análisis factorial. Análisis estadístico multivariable. Santiago de Chile: Editorial Universitaria; 1999. P. 93-39.

25. Richaud MC. Desarrollo del análisis factorial para el estudio de ítems dicotómicos y ordinales. Interdisciplinaria. 2005;22(2):237-51.

26. Abdi H. Factor rotations. En: Lewis-Beck $A B$, Futing T, eds. The SAGE Encyclopedia for research methods for the social sciences. Thousand Oaks, CA: Sage; 2003. Pp. 978-82.

27. Hair JF, Tatham RL, Anderson RE, Black W. Multivariate Data Analysis. 5. ${ }^{\text {a }}$ ed. Chapter 3: Factor analysis. USA: Prentice-Hall; 1998.

28. Mingoti SA. Análise de agrupamentos (Cluster). Análise de dados através de métodos de estadística multivariada. Belo Horizonte: Universidade Federal de Minas Gerais (UFDMG); 2007. P. 295.

29. Hair JF, Tatham RL, Anderson RE, Black W. Multivariate Data Analysis. 5. ${ }^{\mathrm{a}}$ ed. Chapter 9: Cluster analysis. USA: Prentice-Hall; 1998.

30. Li YS, Chuang YC. Neighborhood Effects on an Individual's Health Using Neighborhood Measurements Developed by Factor Analysis and Cluster Analysis. J Urban Health. 2009; 86(1):5-18.

31. Odoi A, Wray R, Emo M, Birch S, Hutchison $\mathrm{B}$, Eyles $\mathrm{J}$, et al. Inequalities in neighbourhood socioeconomic characteristics: potential evidence-base for neighbourhood health planning. Int J Health Geogr. 2005;10(4):20.

32. Carvalho MS, Cruz OG, Nobre FF. Risk profile: multivariate cluster analysis of urban small areas based on socioeconomic indicators-census tracts from the greater Rio de
Janeiro metropolitan area. Cad Saude Publica. 1997;13(4):635-45.

33. Siri JG, Lindblade KA, Rosen DH, Onyango B, Vulule J, Slutsker L, et al. Quantitative urban classification for malaria epidemiology in sub-Saharan Africa. Malar J. 2008;7:34.

34. Macintyre S, Ellaway A. Neighborhoods and health: an overview. En: Kawachi I, Berkman L, eds. Oxford: Oxford University Press; 2003.

35. Harpham T, Grant E, Thomas E. Measuring social capital within health surveys: key issues. Health Policy Plan. 2002;17(1):106-11.

36. Lelieveldt H. Neighbourhood politics: social capital and neighbourhood oriented forms of participation. En: Van Deth JW, Castiglione D, Wolleb G, eds. The Oxford Handbook on Social Capital Oxford. Oxford, US: Oxford University Press; 2006. Pp. 1-27.

37. Gracia E, Herrero J. La comunidad como fuente de apoyo social: evaluación e implicaciones en los ámbitos individual y comunitario. Rev Latinoam Psicol. 2006;38(2):327-42.

38. Winchester L. La dimensión económica de la pobreza y precariedad urbana en las ciudades latinoamericanas. Implicaciones para las políticas del hábitat. EURE. 2008;34(103):27-46.

39. Krannich R, Humphrey C. Using Key Informant Data in Comparative Community Research: An Empirical Assessment. Sociol Methods Res. 1986;14:473-93.

Manuscrito recibido el 17 de julio de 2010. Aceptado para publicación, tras revisión, el 21 de octubre de 2010.

ABSTRACT Objective. To identify residential parameters that characterize the physical and social environment in Chile's irregular settlements, and to construct typologies that will allow to develop profiles with those distinctive residential attributes.

Residential typologies in Chilean irregular settlements with precarious housing conditions

Methods. The study examined the universe of irregular settlements $(n=122)$ in Chile's Metropolitan Region, based on the 2007 national inventory of irregular settlements conducted by the "Un Techo para Chile" foundation. Information about the communities and their locations was obtained from key informants, and these variables were modeled using factor analysis to identify residential parameters, which were then refined by k-means clustering.

Results. The factor analysis pointed to three underlying parameters: local social response, the environment beyond the community, and basic services. The key variables shaping these categories were the existence of housing projects, the urban area involved, and access to electricity. The cluster analysis generated four profiles that combined the three parameters: an urban profile with relatively reliable basic services $(n=30)$, an urban-rural profile with high potential for local social response $(n=32)$, an urban profile with a high level of environmental threat $(n=43)$, and a rural profile with low potential for local social response $(n=17)$.

Conclusions. The residential parameters formed are consistent with the revised theoretical model and suggest relevant indicators for monitoring these communities. Definition of the profiles facilitates assessment of the heterogeneity of residential situations, which helps in the prioritization of areas with deficits or risks that may be present in each cluster. This, in turn, opens the door to further investigation and to exploring opportunities for action.

Key words Human settlements; poverty areas; vulnerable groups; censuses; environmental health; social organization; communitarian organization; Chile. 